This item was submitted to Loughborough's Research Repository by the author.

Items in Figshare are protected by copyright, with all rights reserved, unless otherwise indicated.

\title{
Midwives' thoughts about musculoskeletal disorders with an evaluation of working tasks
}

PLEASE CITE THE PUBLISHED VERSION

https://doi.org/10.1016/j.apergo.2020.103263

PUBLISHER

Elsevier

VERSION

AM (Accepted Manuscript)

\section{PUBLISHER STATEMENT}

This paper was accepted for publication in the journal Applied Ergonomics and the definitive published version is available at https://doi.org/10.1016/j.apergo.2020.103263.

\section{LICENCE}

CC BY-NC-ND 4.0

\section{REPOSITORY RECORD}

Okuyucu, Kubra, Sue Hignett, Diane Gyi, and Angie Doshani. 2020. "Midwives' Thoughts About Musculoskeletal Disorders with an Evaluation of Working Tasks". Loughborough University. https://hdl.handle.net/2134/12865967.v1. 


\section{Midwives' thoughts about musculoskeletal disorders with an evaluation of working tasks}

Kubra Okuyucu; Sue Hignett; Diane Gyi; Angie Doshani

\section{Abstract}

The prevalence of musculoskeletal disorders is very high among midwives resulting in sickness absenteeism, functional limitation and staff shortages. There are several contributory risk factors having impact on the development of MSDs. This study aimed to (1) explore midwives' experiences and views about work-related MSDs and contributing risk factors, and (2) analyse working postures for musculoskeletal injury risks. A mixed method approach was used with interviews/focus group $(n=15 / 7)$ and observations ( $n=22$ ) of specific tasks (during birth and after birth) using the posture analysis observational method (Rapid Entire Body Assessment, REBA). The participants were midwives who had an active role in the United Kingdom (UK) National Health Services (NHS). It was found that MSDs were often attributed to the physical (working in awkward positions), organisational (longer shift hours, fewer staff, increased work load), psychosocial (defensive practice, higher demand) challenges of midwifery. All postures had very high to medium REBA risk levels with action categories indicating that action or further assessment is definitely necessary to reduce MSDs. This research provides a holistic approach by analysing risk factors and interactions in the work context to inform the development of risk management strategies. Midwifery working conditions have a big impact on developing musculoskeletal symptoms. Management of such symptoms will improve staff wellbeing, mother and baby safety, individuals' life trajectories and staff shortages.

\section{Introduction}

Musculoskeletal Disorders (MSDs) are an important issue in midwives. In a recent survey, the prevalence of MSD in UK midwives was found to be $92 \%$ and the most commonly reported body part was the low back (71\%), followed by the neck (45\%) and shoulders (45\%) (Okuyucu et al., 2019). The negative impacts of MSDs have been well described in terms of staff wellbeing, quality of life, job 
satisfaction, economic burden due to sickness-related absences and management procedures, and productivity at work (Long et al., 2013a). Therefore, the consequences of MSD have an effect on midwives, patients and organisations.

In maternity, limitations in productivity or functionality can cost the life of the mother/baby or cause critical and chronic damage. It was reported that 921 babies (out of almost 800,000 ) were lost or had severe brain damage due to something going wrong during labour in 2015 (RCOG, 2016), and 1,123 babies (of nearly 700,000) in 2016 (RCOG, 2018). Maternity claims due to preventable deaths or injuries constitute a significant share (48\%) of the total claims against the NHS, for example the amount of such claims was $£ 2.1$ billion for the period 2017-2018 (NHS Resolution, 2018). In October 2018, the NHS paid $£ 37$ million to a 6-year old boy for causing a catastrophic brain damage due to delayed treatment following his birth (Marshall, 2018).

The exposure to physical working activities has commonly been associated with MSDs in various health professionals, particularly nurses (Lipscomb et al., 2002; Smedley et al., 2003; Smith et al., 2006), surgeons (Szeto et al., 2009), sonographers (Russo et al., 2002), as well as midwives (Long et al., 2013c). Because, a biomechanical imbalance develops when the job demands do not match or fit the capacity of person attempting it (Vanwonterghem et al., 2012; Waters, 2010). This imbalance leads to a high chance of resulting in an injury. The midwifery caring tasks during the birth involve mostly static and/or awkward positions mainly based on the mother's comfort as well as manual handling activities. Excessive and awkward positions in midwifery, for example supporting mother with breast feeding, were agreed to result in potential MSDs (Hignett, 1996; Nowotny-Czupryna et al., 2012).

In the UK over 20 years, mothers have been encouraged to be more mobile during birth including kneeling, standing, sitting and getting into birth pools, unless there is a risk for mother or baby. Moreover, delivering babies is focussed on the woman and her needs/decisions/choices (Cumberlege, 2016). This results in midwives potentially having to adopt awkward positions 
including twisting, extreme neck and back flexion and kneeling; it is hard to avoid these positions in a low-risk birth (where the woman is less likely to be on the bed).

Organisational culture has been linked to healthcare quality and performance (Mannion et al., 2005; Scott et al., 2003), and musculoskeletal health and safety (Hignett, 2001). It is simply defined as 'the way the things are done'; representing the common values, beliefs, attitudes and behavioural norms of the workers, and the effects of tasks, individuals and management strategy (Mannion et al., 2004). Over the last 20 years, midwifery practice has changed with longer shift hours, fewer staff, a higher number of expecting mothers, greater demands by mothers, and more complex births (RCM, 2015). Therefore, organisation-related factors might have a significant impact on the risk of developing MSDs because of the heavier physical and psychological working demand in midwifery. A recent study exploring the prevalence of and risk factors for MSD in UK midwives found that longer working hours was a risk factor for shoulder symptoms, and job stress was related to increasing risk of neck and shoulder symptoms (Okuyucu et al., 2019). An RCM survey study of more than 2,700 midwives exploring the reasons why midwives leave found that they were concerned about making mistakes due to working 12-hour shifts (Leversidge, 2016); 48\% of leavers reported not being satisfied with the quality of care they were able to deliver, and 30\% were not happy with the shift hours.

There are several individual and work-related factors which have been associated with MSDs in healthcare professionals (Long et al., 2012). In order to prevent or reduce MSDs, an ideal management plan should start with understanding the risk factors and then addressing these issues, rather than interventions based on manual handling training (Hignett, 2003; Van Mechelen et al., 1992; Yazdani et al., 2015). Due to wide variations of practice and environment within the healthcare professionals, it is important to address MSD factors and hazards for each profession group. However, to date there has been very limited multifactorial investigation of MSDs specific to maternity professionals that might cause injury. 
To improve the understanding of risk factors for midwives, this research has two aims: (1) to explore midwives' experiences and views about work-related MSDs and contributing risk factors, and to investigate their level of awareness and support by the organisation about safe practice and MSDs prevention strategies; (2) to analyse the most frequent, extreme and working postures with regard to contributing risk level and physical exposure on the musculoskeletal system.

\section{Methods}

A mixed method approach was used. Initially interviews were chosen as an appropriate data collection method to explore issues related to MSDs and explore midwives' experiences and risk factors. A confirmatory focus group was conducted following the completion of the individual interviews to validate the findings and confirm that thematic saturation was achieved. Finally, the risk of MSDs associated with specific working tasks was evaluated using the Rapid Entire Body Assessment (REBA) posture analysis observational method.

\subsection{Procedures}

\subsubsection{Interviews}

An interview schedule (Appendix 1) was developed based on previous literature to explore midwives' perspectives of injury occurrence and risk factors, impact on patient care, support by the organisation, and coping strategies.

The semi-structured interviews covered the following main themes:

I. MSDs and management strategies

II. Support and actions undertaken by the organisation

III. Awareness of health and safety, and prevention strategies

IV. Perception of impact on patient care/safety

V. Identification of working tasks that could be accountable for injuries 
The first theme explored MSDs and link to work. Interviewees were asked to briefly describe their symptoms and their thoughts about whether it was work related. If they did not report any symptoms, they were asked about commonly experienced symptoms by their colleagues and whether they thought that work could be a contributory factor. The previous study (Okuyucu et al., 2019) showed that $92 \%$ of the midwives had experienced MSDs; so interviewees were asked about their views on changes over the last 20 years which might contribute to this high prevalence rates. Interviewees were asked whether they thought ageing and experience in midwifery has an effect on MSDs to explore a correlation found in the previous study (Okuyucu et al., 2019). This theme additionally allowed exploration of management strategies such as self-management or referral to health professionals and the decision process. As reported in literature, short/long term coping strategies were also included (Long et al., 2013b).

The second theme investigated the role of the organisations for MSDs including management and prevention strategies. This theme included the workplace and equipment related issues that might be considered as a risk factor by midwives (Hignett, 1996).

The third theme focussed on exploring the knowledge about prevention strategies at work including self-developed strategies and training. This was included to understand whether midwives protect themselves at work, and if they do, what strategies they use.

The fourth theme explored the impact of MSDs on patient care and safety. The previous study (Okuyucu et al., 2019) showed that musculoskeletal symptoms caused reduction in work and/or leisure activities. Therefore, this theme further explored the impact of midwives' limitations due to MSDs on the caring activities.

The physical work challenges were explored for the next posture analysis study; each interview was asked to identify the most frequent and extreme working tasks and then rate those tasks from 0 to 10 , with 0 representing 'not a challenge at all' and 10 representing 'extremely challenging'. The 
interview schedule concluded with asking interviewees' views about their suggestions to reduce MSDs and to enable continued working in their current role without injuries.

An invitation email was sent to all the midwives, irrespective whether they had experienced any MSDs or not, via the Head of the Midwifery at University Hospitals of Leicester and the Consultant Midwifery UK network to recruit participants for the study by contacting the researcher to arrange a time and place for the interview.

Some of the interviewees were identified and invited to join the study by other interviewees.. Additionally, midwives who were interested but who could not attend the interviews were encouraged to participate via telephone and email interviews.

\subsubsection{Data collection}

Before starting the interview, the overall study and information about the objective of the interview was introduced including confidentiality and anonymity. Consent was received for the interview to be recorded using a voice recorder. Demographic details were collected such as age, working status, year of experience and place of working. As the nature of semi-structured interviews, the questions were not restricted to the order in the schedule, it allows the discussion to flow naturally and freely (Robson and McCartan, 2016). Prompts were developed to encourage the interviewees to provide more depth in their responses, such as 'can you tell me more about that?' and 'can you give me an example of that?' Interviewees' names were not used at any stage of the data collection process. Duration of the interviews was 13-56 minutes.

\subsubsection{Data management and analysis}

This study uses a qualitative approach as a flexible study design based on grounded theory. The main feature of this approach is to develop theories while continuing data collection and analysis based on the theories generated (theoretical sampling). Therefore, it was a dynamic process: it started with field work then carried on analysing and generating theories; and then returned back to 
collecting more data based on the previous analysis; and etc. This process ended when there was no new knowledge added on the existing categories (theoretical saturation). Eight midwives were interviewed in the first-round of the interviews, with a further seven interviews in the second-round. A total of 15 midwives participated in the semi-structured interviews, with a mean age of 44.8 $(S D=10.42$, range $24-62)$ and $15.8(S D=11.02$, range 1-44) years of experience (Table 1$)$. The participants had a wide range of diversity in terms of MSDs, age, experience and roles in midwifery. It is suggested that this adds to the external validity of this study by supporting potential transferability. Four of the total 15 interviews were carried out by telephone.

Table 1: Demographic and employment characteristics of the interviewees $(n=15)$

\begin{tabular}{|c|c|c|c|c|c|c|}
\hline $\begin{array}{l}\text { Interviewee } \\
\text { number }\end{array}$ & Age & MSDs & $\begin{array}{l}\text { Age at first } \\
\text { symptoms }\end{array}$ & $\begin{array}{l}\text { Practice } \\
\text { year }\end{array}$ & Current role & $\begin{array}{l}\text { Work } \\
\text { pattern }\end{array}$ \\
\hline 1 & 46 & Low back & 34 & 15 & Midwife & Full time \\
\hline 2 & 38 & No & $\mathrm{N} / \mathrm{A}$ & 8 & Midwife & Full time \\
\hline 3 & 50 & Low back & 19 & $\begin{array}{l}21 \text { years } \\
\text { clinical+4 } \\
\text { years } \\
\text { educator }\end{array}$ & $\begin{array}{l}\text { Education and Practice Development } \\
\text { Midwife }\end{array}$ & Full time \\
\hline 4 & 43 & Low back & 20 & 23 & Divisional Risk Management Midwife & Full time \\
\hline 5 & 62 & Low back & 39 & 44 & Midwife - mostly antenatal clinic & Part time \\
\hline 6 & 41 & Knee & 38 & 12 & Midwife & Part time \\
\hline 7 & 31 & Back & 27 & 4 & Midwife - Band 6 & Full time \\
\hline 8 & 34 & Back & 14 & 11 & Midwife & Full time \\
\hline 9 & 58 & $\begin{array}{l}\text { Back, } \\
\text { Wrist }\end{array}$ & 56 & 10 & Midwife - Band 7 coordinator & Full time \\
\hline 10 & 51 & $\begin{array}{l}\text { Wrist, } \\
\text { Shoulders, } \\
\text { Low back }\end{array}$ & 45 & 12 & Midwife coordinator & Part time \\
\hline 11 & 37 & No & $\mathrm{N} / \mathrm{A}$ & 1 & Midwife & Full time \\
\hline 12 & 50 & Back & 35 & 25 & Midwife + corporate role & Full time \\
\hline 13 & 24 & Knee & 22 & 2 & Midwife & Full time \\
\hline 14 & 50 & Shoulder & 40 & 25 & Midwife & Full time \\
\hline 15 & 58 & No & $\mathrm{N} / \mathrm{A}$ & 21 & Senior midwife - matron & Full time \\
\hline
\end{tabular}

The recorded interviews were allocated a unique identifying number (e.g., M01, M02, M03...) to name audio files and transcript documents. Audio recordings were listened to carefully, transcribed into Microsoft Word 2010 and exported to Nvivo11, a qualitative data management software tool for coding and analysis. The recordings were transcribed verbatim, and non-verbal expressions were noted during the interviews. 
Transcripts were coded using a thematic coding approach, in which the data are coded and labelled according to similar interests, then the codes with the same labels are grouped together as a theme. Additionally, a quasi-statistical approach was used to determine the importance of the theme based on the frequency of words or phrases, for example identification of work challenges.

\subsubsection{Confirmatory focus group}

The confirmatory focus group recruited a new sample of midwives who were not the original interviewees to provide an opportunity to assess transferability of the findings (LoBiondo-Wood and Haber, 2006). Seven midwives participated in the focus group, with a mean age of 40.7 (SD=11.89, range $27-55)$ and $13.5(S D=7.8$, range $5-28$ ) years of experience (Table 2 ).

Table 2: Interviewee characteristics of the focus group ( $n=7)$

\begin{tabular}{|l|l|l|l|l|l|}
\hline $\begin{array}{l}\text { Interviewee } \\
\text { number }\end{array}$ & Age & MSDs & $\begin{array}{l}\text { Practice } \\
\text { year }\end{array}$ & Current role & Work pattern \\
\hline $\mathbf{1}$ & 55 & $\begin{array}{l}\text { Low back } \\
\text { Knee } \\
\text { Shoulder }\end{array}$ & 17 & Community Midwife & Full time \\
\hline $\mathbf{2}$ & 51 & $\begin{array}{l}\text { Previous } \\
\text { knee injury }\end{array}$ & 17 & $\begin{array}{l}\text { Deputy manager maternity } \\
\text { assessment unit midwife }\end{array}$ & Full time \\
\hline $\mathbf{3}$ & 52 & No & 28 & $\begin{array}{l}\text { Community midwife team lead } \\
\text { Community Midwife }\end{array}$ & Full time \\
\hline $\mathbf{4}$ & 37 & No & 11 & Part time \\
\hline $\mathbf{5}$ & 27 & No & 7 & Midwife on ward & Full time \\
\hline $\mathbf{6}$ & 27 & No & 5 & Midwife - Band 6 & Full time \\
\hline $\mathbf{7}$ & 36 & No & 10 & Midwife & Part time \\
\hline
\end{tabular}

A focus group question guide was produced including the main questions in the initial interviews.

\subsubsection{Posture analysis}

Finally, Rapid Entire Body Assessment (REBA) was used to assess the working postures and exposure to work activity related risk factors. REBA is a postural analysis tool developed by Hignett and McAtamney (2000) as a postural analysis tool that is sensitive for assessing postures adapted in healthcare with recording dynamic, static and rapidly changing postures; applied force/load; and handling of the load with hands or another part of the body.

Each body part (except the foot and ankle) is scored individually according to the position. Initially two scores are obtained: Score A and Score B, for the analysis of neck, trunk and legs (Score A); and the arm and wrist (Score B). The final REBA score shows the level of risk as: Negligible (1), Low (2-3), 
Medium (4-7), High (8-10), or Very High (11-15). Event or time-based sampling techniques can be used to select the postures for assessment. Event sampling was utilised in this study to analyse the most common, extreme and/or awkward targeted postures with high sensitivity and less complexity.

An open invitation was sent to midwives with an active clinical role at Leicester General Hospital through the Head of the Midwifery and the Lead Consultant of the Birth Suite. Snowball sampling was also used for recruitment during the data collection, over a 5-day period with 22 midwives volunteering to take part in the study (Table 3).

Table 3: Characteristics of the participants for posture analysis $(n=22)$

\begin{tabular}{|c|c|c|c|}
\hline Characteristics & $\underline{n}(\%)$ & mean (sd) & Range \\
\hline Age & N/A & $34.73(8.4)$ & $25-58$ \\
\hline Height (m) & N/A & $1.68(0.8)$ & $1.48-1.80$ \\
\hline Weight (kg) & N/A & $71.36(18.6)$ & $45-130$ \\
\hline BMI $\left(\mathrm{kg} / \mathrm{m}^{2}\right)$ & N/A & $25.1(6.3)$ & $15.6-48.3$ \\
\hline $\begin{array}{l}\text { Dominant hand } \\
\text { Right } \\
\text { Left }\end{array}$ & $\begin{array}{l}19(86.4) \text { n (\%) } \\
3(13.6) \text { n (\%) }\end{array}$ & & N/A \\
\hline Years practicing in midwifery & N/A & $6.41(6.02)$ & $1-21$ \\
\hline $\begin{array}{l}\text { Work pattern } \\
\text { Full time } \\
\text { Part time }\end{array}$ & $\begin{array}{l}18(81.8) \text { n (\%) } \\
4(18.2) \text { n (\%) }\end{array}$ & & N/A \\
\hline
\end{tabular}

\subsubsection{The setting}

Due to the unpredictability of collecting data during observed practice in real clinical scenarios, it was decided to analyse simulations of the specified tasks. The main activities were the same, and the data could be collected without distractions. The tasks were performed by the participants with a model (a woman acting as a mother) in a birth room to give the same environment and equipment as real practice. The participants were encouraged to carry out the tasks the way they felt comfortable in a real practice. The data were recorded by three cameras from different angles (Figure 1). The cameras were moved depending on the position of the participant during the task. 


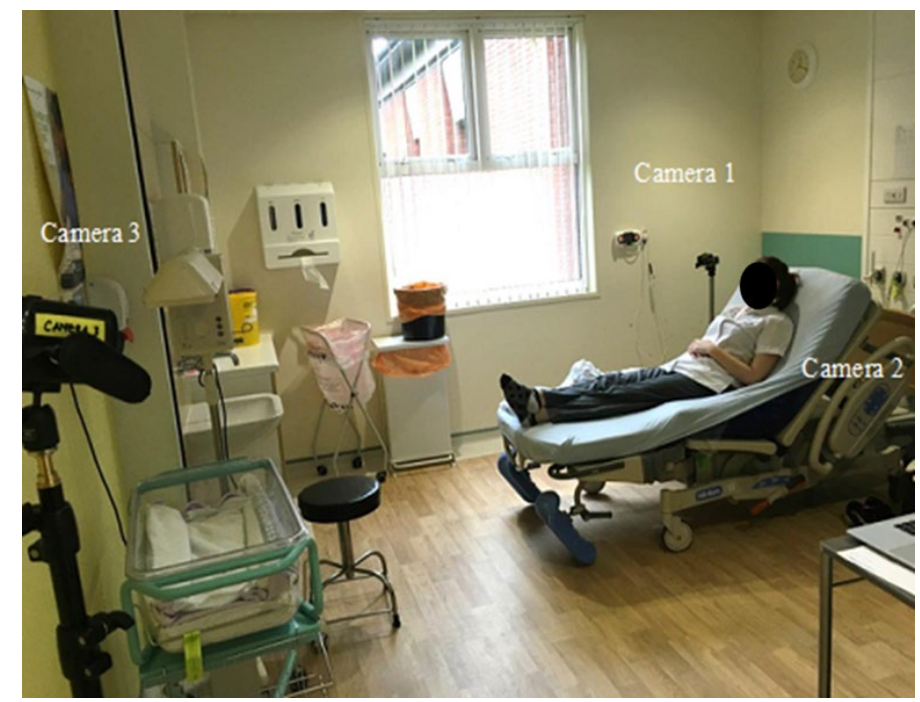

Figure 1: Layout of birth room and position of cameras

\subsubsection{Selecting the postures for assessment}

The most frequent, extreme or awkward working tasks, and those causing discomfort were identified from the interviews.

The highly rated top four tasks were:

- $\quad$ Vaginal examination (VE) /Artificial Rupture of Membrane (ARM)

- $\quad$ Birth positions with mother standing or kneeling

- $\quad$ Suturing, including:

o Placing the mothers' legs in lithotomy

o Taking out the end of the bed and putting it back in

- $\quad$ Supporting breast-feeding

These tasks were divided into 9 tasks for analysis. Table 4 presents the description and reason for selection with example pictures.

Table 4: Tasks chosen for analysis with description and reason for selection

$\begin{array}{lll}\text { No Picture } & \text { Description } & \begin{array}{l}\text { Reason for } \\ \text { selection }\end{array} \\ & \text { Vaginal examination (VE) / ARM } & \text { Reported to } \\ & \text { VE is an essential care activity that } & \text { cause } \\ & \text { midwives involved frequently to assess discomfort } \\ & \text { the progress of labour. In early stages of } & \text { dabour, ARM can be applied to induce }\end{array}$




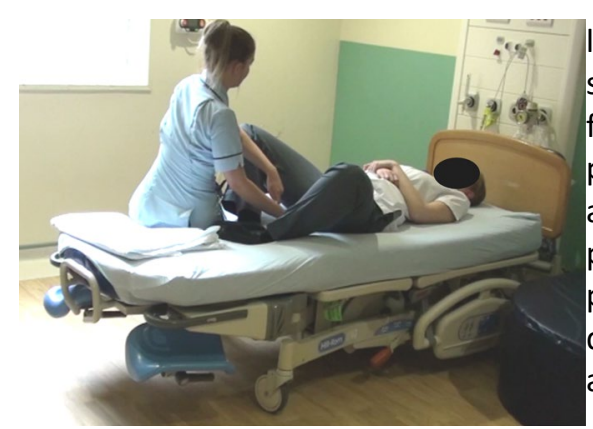

labour, following VE. A midwife commonly sits on the edge of the bed and turns her face to the mother and carries out the procedure using both hands with an amnihook (if necessary), as shown in the picture. ARM is applied only once and the procedure lasts about 10 minutes depending on the mother's cooperation and comfort.

2

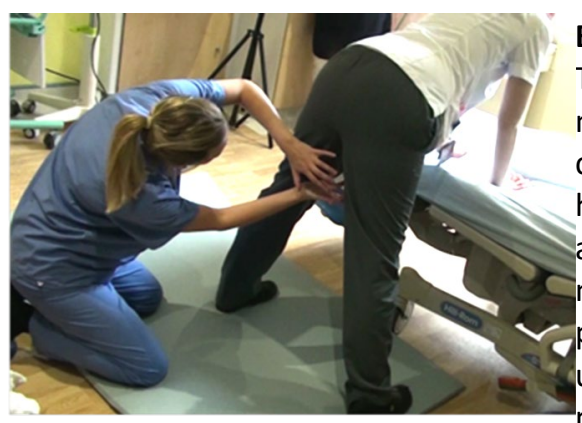

Birth positions with mother standing

This task involves a midwife caring with a mother in standing labour position. The caring activities include listening the fetal heart (auscultation), vaginal examination and grabbing the new born baby. A midwife commonly carries out the procedures on the floor with bending and upper arm flexed and/or abducted position. The overall labour duration is unpredictable; however a midwife is in this position to monitor fetal heart rate or vaginal examination once every 15 minutes for 60 seconds in the first stage; after every contraction or every 5 minutes in the second stage of the labour.

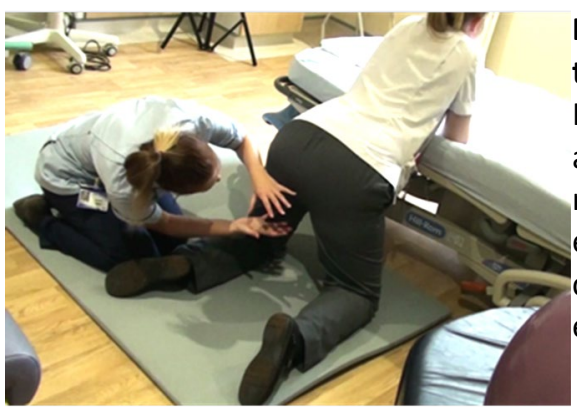

Birth positions with mother kneeling on the floor

In this position, a mother is in a kneeling and bending forward position, and a midwife is mostly on her knees with excessive trunk flexion and twisting to carry out the procedures at least once every 15 minutes.

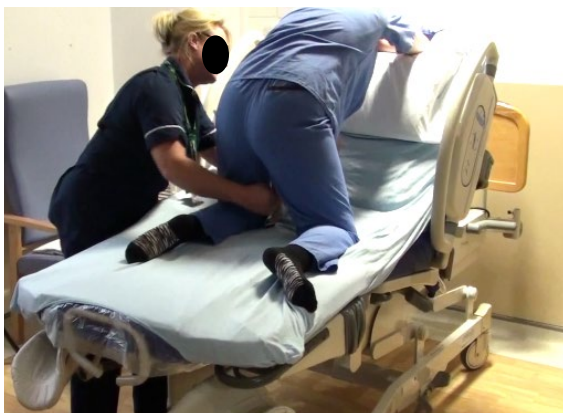

Birth positions with mother kneeling on the bed

A mother can kneel on the bed during the labour, so a midwife works in standing position. She carries out regular vaginal examination, auscultation and grabs the new born baby in this position with bending over to reach and see. The frequency varies, but a midwife gets this position at least once every 15 minutes for caring activities.
Reported to cause discomfort

Extreme, awkward

Reported to cause discomfort

Extreme, awkward

Reported to cause discomfort 


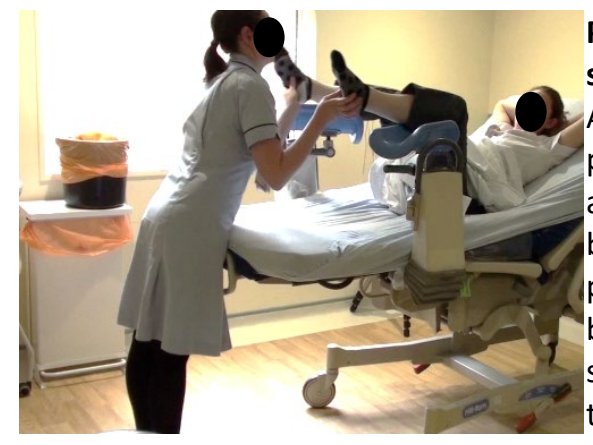

Placing the legs into lithotomy before suturing

A midwife starts to prepare a mother for perineal suturing after labour. In this task, a midwife lifts a mother's legs lying on the bed and places them into lithotomy. This picture shows a midwife grabbing the both legs from the ankles and lifting at the same time with trunk flexion. Considering the average weight of a leg to be $12 \mathrm{~kg}$ and mother not being cooperated or tired after birth, this task requires a great muscle force.

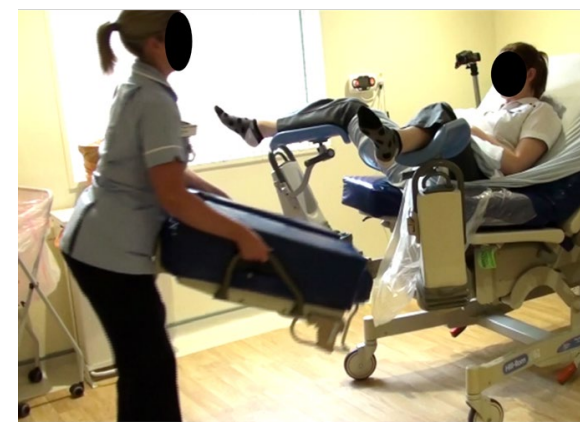

Detaching the end of the bed before suturing

The bed needs to be prepared for suturing process. After placing the legs into lithotomy, the end of the bed (with a separate mattress part) is taken out in order to get closer. The detachable part in this picture weighs around $6 \mathrm{~kg}$. A midwife lifts and then places it on the floor to attach it later.

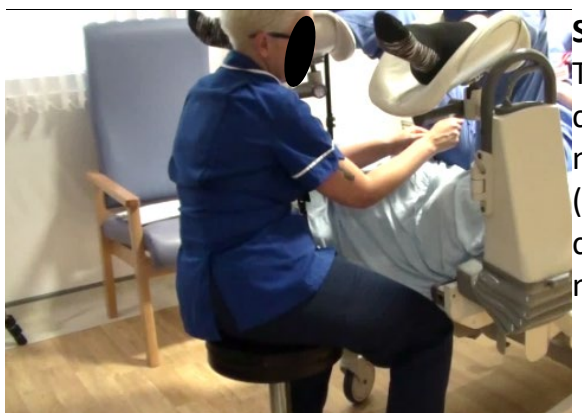

Suturing process

This task requires midwives to sit on a chair, get the equipment ready on a tray near them, place the source of light (mostly left/right back of the midwife) and carry out the procedure, which lasts 20-30 minutes.

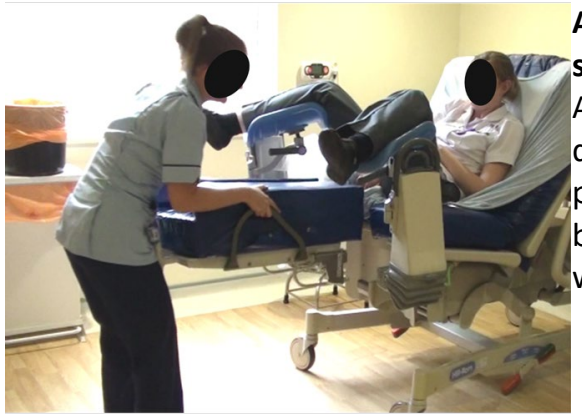

Attaching the end of the bed after After the completion of the suturing, the detachable part is lifted from the floor and placed into the attachment points in the bed. The detachable part in this picture weights around $6 \mathrm{~kg}$.

Reported to cause

Reported to cause discomfort

Sustained long duration

discomfort 


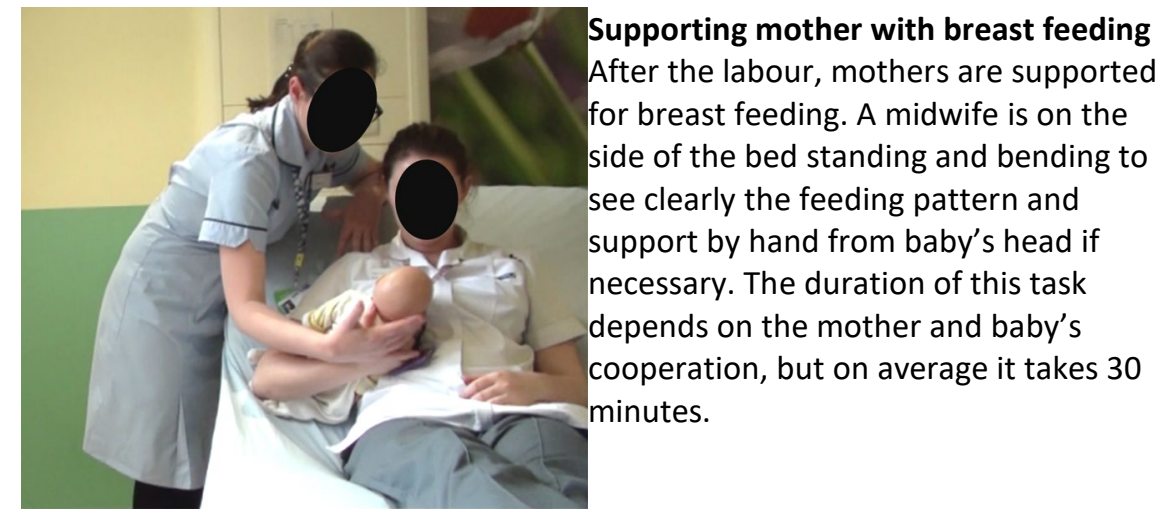

Reported to

cause discomfort

Sustained long duration

Participants carried out these tasks in sequence, starting with vaginal examination and ending with supporting a mother with breast-feeding. The simulation of the tasks lasted 30-40 minutes, including the setting changes before each task. At the end of the tasks, each participant completed a questionnaire with questions about their demographics (age, height, weight and dominant hand) and work situation (clinical experience, work pattern).

The most extreme postures adopted by participants during the tasks were extracted from the video data, with event sampling. Three-camera records ensured the best view to measure the range of motion. An inter-rater reliability exercise was utilised to ensure the accuracy of the researcher in assessing the imaging using REBA. Approximately $7 \%$ of the data ( $n=10$ postures) were assessed and rated independently by 14 trained raters (including the researcher). The level of agreement between the 'raters' was quantified by using Intraclass Correlation Coefficient (ICC) for the final REBA scores. The single measure was found 0.779 , which is 'good' according to the interpretation of values (Koo and Li, 2016).

A score was assigned according to REBA analysis with the measured range of motions. In addition, twisting or side flexion of the trunk and neck; weight bearing on the legs; abduction, rotation, shoulder elevation, support or gravity assistance of the upper arms; and deviation or twisting of the wrist were scored. The position of grip, load being carried and the activity were also considered and rated. Then, the scores for each part were combined to generate a final REBA score indicating the 
degree of MSDs risk. Descriptive statistical tests were used to present the summary of demographics and REBA scores.

The research received ethical approval from Loughborough University Ethics committee. Additional ethical clearance required by UK Health Research Authority and Local Trust (University Hospitals of Leicester) was also granted.

\section{Findings}

\subsection{Interviews}

\subsubsection{MSDS}

Of those interviewed, $80 \%(n=12)$ reported having one or more MSDs with the back being the most affected area $(n=8,53 \%)$. They also suggested that shoulder and knee symptoms were quite common among midwives. The interviewees were unanimous in the view that the symptoms were due to work related activities and/or that their current injuries were aggravated by working tasks. For example, back symptoms were thought to be due to assisting breast feeding or the positions for internal examination e.g., sitting on edge of the bed, turning and twisting to access the woman. Knee symptoms were thought to be due to supporting the mother on the floor; "My knees, from being kneeling - specifically from looking after a few labours in a room and I was on the floor" (M07). Many of the interviewees were reluctant to ask for sick leave and concerns were expressed about taking sick leave. For example, one interviewee stated that if she takes sick leave, her colleagues will be overloaded to cover her absence. A small number of interviewees had been referred to Occupational Health Department due to their injuries. However, many midwives did not report their problems because they did not feel that they would get benefit from reporting and the subsequent management procedures.

Limitations in normal activities at work or outside the work were mentioned by many of the interviewees. They also expressed their feelings about being restricted at work due to their symptoms, and not being as active and healthy as they used to be before their injuries; 
"I go very slowly. Everything just takes longer, so the women are waiting longer." (M01)

"There is no way I will crawl around the floor or bend over for pool birth or something." (M03).

Some midwives argued that their symptoms did not influence patient safety because they prioritised mothers. On the other hand, the interviewees supported the idea that it could have an impact on patient care and safety; "...my capabilities would be reduced, therefore risking my patient." (M13). The interviewees mostly agreed that each midwife needs to be responsible for protecting themselves from potential injuries. This responsibility included either being aware of the hazards as part of the job and using your body properly and/or using the equipment or external support efficiently to minimise the force. However, many indicated that due to the heavy workload it was not always possible to think about all of these things (e.g., adjustment of equipment, thinking about their own position and adapting for the most suitable posture).

With respect to training, all interviewees reported that they had done a standardised mandatory manual handling e-learning training course every year (which used to previously be run face to face). Some Trusts also organised clinical training days 2-3 times in a year. They all commented on the content of training, and the majority $(n=11)$ agreed that the training was not specific to midwifery related tasks or positions, rather it was general for mostly nursing manual lifting activities. Therefore, the training does not provide support or advice for common midwifery working activities.

\subsubsection{Organisation}

They all agreed that not having enough breaks during shifts, longer shift hours, fewer staff and increased work load led to gradual exhaustion. This could also result in dehydration and irregular eating patterns, which would impact on staff well-being; "I have my breakfast at 6.00 and I don't normally have my lunch before 17.00."(M08). The workload caused them to skip ordinary tasks due to time pressure which would protect them such as raising the bed; "We just do things to save time, because we are so busy - we don't think about ourselves, we just do it automatically." (M04) Some believed that training was given by the organisation instead of improving conditions, as indicated; "There is a lot of stuff could be better is not necessarily provided by the Trust either, but 
you are expected still to give that care because like I said you can't deny woman - that choice."

(M02). Although there have been actions by the organisations, there was felt to be slow progress, as one interviewee quoted; "...anything in the NHS takes an age to sort of. It is like dragging a lumber of dinosaur behind you." (M10)

\subsubsection{Midwifery}

Many acknowledged the physical and psychological demands of midwifery care. There are certain things that they believe are the nature of the work and they cannot be changed.

1) Caring for more than one patient (mother and baby) at a same time; "I am aware that sometimes we have two or three lives in our hands in one room." (M07),

2) Working autonomously; so it is very rare that they ask for help,

3) Unpredictability of cases,

4) Being positioned depending on the mother's preferences, and staying there sometimes for a long time,

5) Midwives care for mobile mothers with regular examinations off bed, on birthing ball or pool;

"...women are encouraged to remain mobile in labour and adopt different positions. This means midwives are having to get into awkward positions themselves." (M09),

6) Continuously changing positions.

Due to the factors listed above, the interviewees thought that having any pain was an inevitable consequence in their occupation.

Two of the interviewees commented on the psychological challenges of the midwifery work. One thought that encouraging mothers during birth is more exhausting than physical work.

\subsubsection{Staff}

This theme related injury occurrence to the role of midwives' characteristics or attitudes. A variety of perspectives were expressed mainly in relation to age and individual attributes.

Some interviewees thought that ageing had a detrimental effect with older midwives tending to have more injuries, while others argued that younger ones were more likely to get injury for 
different reasons. For example, interviewees commented that younger colleagues can feel empathy with the mothers' preferences, but older midwives are able to negotiate a suitable compromise. This also reflects feeling less confident to say that they have limitations to support a certain position; "[They (younger midwives) feel like] I can't talk and hurt myself, because that is what I want to do for the benefit of the patient." (M10).

It was mentioned by many interviewees that reduced manual handling training and not having classbased, face to face training, may have contributed to newly qualified midwives injuring themselves. It was also suggested that having experience helps midwives know how best to look after themselves. Another reported reason was limited knowledge of their rights in an organisation, so younger midwives were worried about complaints. On the other hand, one interviewee suggested that the older generation could handle pain more than younger generation.

A key theme from the interviews was concern about complaints from mothers which could result in a culture where midwives practice defensively; "I think we tend to accept that what the patient wants the patient gets because should the patient then complain we would be seem to be fault." (M10). Defensive practice may lead midwives to do much more than they should. Personal behaviours and attributes of staff were agreed to have an impact on injuries. It was an agreed concern that prioritising the patient and not caring for themselves were common faults. However, many indicated that they could not put the mother second; "We are all guilty of trying to do the best for woman that we are looking after. We are thinking of 'ohh really, she should not use me', but all about the baby is coming it is fine." (M02). "Midwives are in a very caring nature, I think the individual that goes forward is in midwife - it is that caring nature." (M12)

\subsubsection{Patients}

A common view amongst interviewees was that patient-related factors had a key role on the occurrence of injuries. Many of the interviewees commented on the burden of caring for high BMI mothers during tasks such as moving to/on the beds and getting their legs in the lithotomy position. 
"Some women would like to go on the floor - I really really struggle. But because you want to let the woman what she wants to do, you just do it." (M04). "You can't deny somebody using the pool because of your back." (M02). These quotes illustrate the interviewees' views on the impact of mothers' choice of birth positions. However, the majority of the interviewees reported that they supported the mothers in their choice of positions despite their own discomfort. The views about mothers' expectations varied. Many interviewees expressed their opinion that recently mothers are requiring more support compared to the past, which may be due to the increase in epidurals and caesarean section (C-section), making them less mobile and needing more care.

Most of the themes from the initial interviews were addressed in the focus group; there were no new themes. Therefore, it was confirmed that the saturation of the themes had been achieved and the results had been validated.

\subsection{Posture analysis}

From the analysis of working postures, a total of 140 positions were identified from the video recordings. Table 5 shows the number of participants performed each task and the analysis of the postures using the REBA worksheet.

Table 5: Postural analysis results with REBA scores

\begin{tabular}{|c|c|c|c|c|c|c|c|}
\hline $\begin{array}{l}\text { Task } \\
\text { no }\end{array}$ & $\begin{array}{l}\text { Number of } \\
\text { participants } \\
\text { performed the } \\
\text { task }\end{array}$ & $\begin{array}{l}\text { Body parts } \\
\text { in the most } \\
\text { extreme } \\
\text { position }\end{array}$ & $\begin{array}{l}\text { Mean } \\
\text { REBA } \\
\text { score } \\
\text { (SD) }\end{array}$ & Range & $\begin{array}{l}\text { Action } \\
\text { level }\end{array}$ & $\begin{array}{l}\text { Risk } \\
\text { level }\end{array}$ & Action \\
\hline 1 & 20 & $\begin{array}{l}\text { Trunk } \\
\text { Neck } \\
\text { Wrist }\end{array}$ & $7.2(1.2)$ & $6-9$ & 2 & Medium & Necessary \\
\hline 2 & 17 & $\begin{array}{l}\text { Legs } \\
\text { Trunk } \\
\text { Neck }\end{array}$ & $10.2(1.2)$ & $8-12$ & 3 & High & $\begin{array}{l}\text { Necessary } \\
\text { soon }\end{array}$ \\
\hline 3 & 15 & $\begin{array}{l}\text { Legs } \\
\text { Trunk } \\
\text { Neck }\end{array}$ & $10.8(0.7)$ & $9-12$ & 4 & Very high & $\begin{array}{l}\text { Necessary } \\
\text { NOW }\end{array}$ \\
\hline 4 & 12 & $\begin{array}{l}\text { Trunk } \\
\text { Neck }\end{array}$ & $7.9(1.8)$ & $5-11$ & 3 & High & $\begin{array}{l}\text { Necessary } \\
\text { soon }\end{array}$ \\
\hline 5 & 14 & $\begin{array}{l}\text { Upper-arm } \\
\text { Trunk }\end{array}$ & $6.6(1.2)$ & $5-9$ & 2 & Medium & Necessary \\
\hline 6 & 16 & Trunk & $5.3(2.2)$ & $3-9$ & 2 & Medium & Necessary \\
\hline 7 & 14 & $\begin{array}{l}\text { Trunk } \\
\text { Neck }\end{array}$ & $4.8(1.1)$ & $2-7$ & 2 & Medium & Necessary \\
\hline 8 & 15 & Trunk & $5.6(1.3)$ & $3-7$ & 2 & Medium & Necessary \\
\hline
\end{tabular}




\begin{tabular}{|l|l|l|l|l|l|l|l|}
\hline \multicolumn{9}{|c|}{} & Neck & & & \\
\hline 9 & 17 & $\begin{array}{l}\text { Trunk } \\
\text { Neck }\end{array}$ & $5.8(1.6)$ & $4-10$ & 2 & Medium & Necessary \\
\hline
\end{tabular}

The trunk is the most affected body part in all the observed tasks, followed by the neck. Trunk and neck positions were extreme with movement of $>20^{\circ}$ flexion/extension mostly with twisting/side flexion. The upper arm postures were extreme when placing the mothers' legs into lithotomy, because they were mostly $>45^{\circ}$ flexed and abducted position, with shoulders sometimes elevated. The wrist was mostly affected during vaginal examination as the wrist position was $>15^{\circ}$ flexion/extension with deviation. Floor level birth positions had the highest risk for midwives. The legs (particularly knees) were mostly affected in these positions; the weight bearing on legs was bilateral, but knees were mostly $>60^{\circ}$ flexed position. The amount of time in this position is unpredictable; so the midwives kneel during the labour (maybe 1-2 hours) or for examination. Overall, the REBA action levels were $\geq 2$ indicating that changes are definitely necessary to reduce MSDs for midwifery specific working postures.

The REBA scores varied for the same task performed by different midwives. It was also observed that there were slight differences in practice which were recorded in the REBA scoring. This indicates that midwives' characteristics (e.g., age, height, weight), training levels and ability using the equipment might impact on the risk of MSDs.

\section{Discussion}

This research builds on a recent work demonstrating high prevalence of MSDs among midwives resulting in sickness absenteeism, reduction in normal work activities and staff shortages (Okuyucu et al., 2019). This study provides an exploration of risk factors from midwives' perspective and an evaluation of working positions. When considered together, these data provide a holistic perspective by considering all aspects of systems and interactions with midwives at the centre in a work context (Wilson and Sharples, 2015).The strength of this study comes from its comprehensive approach. 
One interesting factor expressed by the midwives as having an impact on developing MSDs was defensive practice. There was concern about the complaints from patients, which creates an environment where midwives practice in a defensive manner. Defensive practice has previously been discussed among health professionals, linked to poor staff health, both physically and psychologically, and impact on patient safety (Passmore and Leung, 2002; Surtees, 2010; Symon, 2000). A study of over 2,000 UK midwives and obstetricians (Symon, 2000) showed that defensive practice resulted in changes in maternity clinical practice, with performing more caesarean sections, more often monitoring, more investigation and more documentation but not necessarily an increase in the quality of care. This can lead them doing much more than they should, with increased muscle strain or adoption of awkward positions and could also be linked to hesitation in clinical practice and poor communication with patients and their relatives (Camerino et al., 2008), particularly among younger midwives, as they may have a greater fear of litigation and will do what the women requests, even when they should not; younger midwives have less experience in negotiation with the mother about positions and/or her requirements, and awareness of their working rights within the organisation. This agrees with the findings of a study of midwives in England conducted over 20 years ago (Hignett, 1996).

Another interesting finding found in this study was midwives having little tendency to report MSDs at work or request sick leave, and therefore they continued attendance while not feeling well (sickness presenteeism) despite limitations. This was confirmed by previous studies (Long et al., 2013a; Okuyucu et al., 2019), as sick leave prevalence was far lower than the prevalence of MSDs and its severity (limitation in normal activities). The reason for not taking sick leave was suggested to be related to feeling overly responsible for patients and colleagues (feeling guilty about their colleagues covering their absence). So, they are motivated to go to work due to team responsibility (Bierla et al., 2013), emotional attachment and sympathy to the patients (Tveten and Morken, 2015). Sickness absence data has generally been used as a measure of health status at workplaces (Black and Frost, 2011; Health and Social Care Information Centre, 2015). Recently, sickness presenteeism 
has also been widely accepted as an indicator of the health state, despite the fact that it is difficult to measure reliably (e.g., number of days unwell at work and/or productivity) (Whysall et al., 2018). It is particularly common in healthcare compared to other sectors (Aronsson et al., 2000; Plant and Coombes, 2003). The potential detrimental effects of presenteeism for both individuals and organisations have been well documented in literature. It is connected to work disability/instability (WI), which is defined as the inconsistency between staff functional or cognitive ability and work demands (Gilworth et al., 2003). Additionally, a systematic review of longitudinal studies showed that this is a risk factor for future sickness absence and self-reported health issues (Skagen and Collins, 2016) and that can influence worker productivity and safety at work (Aysun and Bayram, 2017). All these impacts can lead to higher economic costs (e.g., medication, physiotherapy), in addition to indirect costs (e.g., use of agency staff to fill a vacant shift) (Bergström et al., 2009). Most interviewees attributed their symptoms to static or awkward positions during birth and assisting mother with breast feeding, similar to the reported physical demands for Australian midwives (Long et al., 2013c). Trunk and neck positions were observed to be affected in all midwives' postures with a range of motion mostly $>20^{\circ}$ and twisted or lateral flexed positions. This result is consistent with that of Nowotny-Czupryna et al. (2012) who found the lower back to be the most affected body part (the second was neck) during a basic birth position with the mother is in supine position on the bed. Although many attributed their symptoms to specific working tasks, the origin of injuries at work also varied from patient-related to staff attributes or organisation related factors.

Another finding from this study supports the previous result of midwives' attribution of their symptoms to physical working demand, which is midwives being exposed to heavy workload and stress which constitutes a great biopsychosocial burden on them. This finding agrees with the literature and confirms that heavy workload results in midwives leaving the profession and precludes them from providing women-centred care (Hildingsson et al., 2013; Lundgren et al., 2019) 
or "real midwifery" (O'Connell and Downe, 2009). In the UK, two 12-hour shifts were introduced and recommended for National Health Services (NHS) staff instead of three 8-hour shifts in 1990s. The application of 12-hour shifts increased for nursing staff after 2010 (Ball et al., 2014). Midwives also started working 12.5 hours to incorporate the 12 -hour shift regulation depending on local organisational requests. It has been suggested that some might find this beneficial as working longer days results in fewer number of days worked per week. However longer shifts with fewer breaks have been argued to decrease productivity and increase opportunities for errors (Griffiths et al., 2014; Health Safety Executive, 2006; Rogers et al., 2004).

The interview findings showed that midwives were aware of risks and prevention strategies and that they had a responsibility to protect themselves, but they expressed the idea that it was not always possible to think about themselves while working and to apply these methods in practice. This also linked to the postural analysis findings with all postures being classified at very high to medium risk levels with action categories indicating that action or further assessment is definitely necessary. This correlates with evidence from a recent systematic review (Hogan et al., 2014) that workers' reports of understanding and awareness levels do not always result in behavioural changes in practice. These findings may also support the conclusion that another factor having impact on MSD is the lack of user friendly guidelines to improve implementation in practice (Fallentin et al., 2000). For example, manual handling training sessions are mostly targeted towards nursing-related tasks, such as transferring a patient from bed to bed, bed to chair, sit to stand; use of hoists; safe lifting principles (keeping the object close to the body, less bending instead kneeling). Almost all interviewees agreed that they did not benefit from this compulsory generic training for reducing their MSDs risk. This is consistent with previous systematic reviews (Hignett, 2003; Dawson et al., 2007; Clemes et al., 2009; Verbeek et al., 2011; Hogan et al., 2014) which suggest that manual handling training has no impact on reducing MSDs. This might be because manual handling training is rarely tailored to a particular occupation or task. For example, McDermott et al. (2012) investigated the effectiveness of manual handling training practices with 120 organisations and 30 
training consultancies, finding that the majority of organisations used classroom-based generic training comprising non-specific tasks. It was suggested that for manual handling training to be effective it should be task- and occupation-specific. For example, Hignett (1996) suggested over 20 years ago that manual handling tasks for midwifery differ from nursing care mainly due to having two 'loads' (mother and baby) and caring for healthy mobile 'patients'. It should also be noted that the equipment and environment is unique for midwifery such as birthing beds and pool. All these factors would change the nature of the manual handling operations.

Overall, prevention of work-related MSDs may change individuals' life trajectories. Concerning MSD, there is a need for an understanding and evaluation of human interactions with other elements of a system, because "It is often easier to change things around people than to change people themselves". (CIEHF, 2018). Even though individuals have a responsibility to maintain safe practices in order to have longer working lives, it is an organisational responsibility to provide for the needs of each worker and create more favourable environments for its ageing workforce. Considering all the positive and negative effects of ageing at work, individuals should be empowered to work as long as they wish to. To enable this, suggestions may include more flexibility in working hours, employing additional staff, more rotations to allow staff to spread their workloads, assessing physical and psychological working demands, redesigning the working environment when necessary, more occupation-tailored manual handling training and increasing the awareness of workers' rights within an organisation.

\subsection{Methodological considerations}

A mixed method approach was applied in this study to enable a comprehensive understanding of the issues and therefore to develop more targeted strategies. In the absence of any real data being reported in relation to this issue over the last 20 years, gaining an essential understanding of risk factors via different methods is critical to the development of management strategies to reduce MSD in this occupational group. However, there are some considerations about the methods. 
Although participation was open to all midwives having a role in the UK maternity services, the interviewees who responded were mostly from one Trust which might make the findings less generalisable to all midwives in the UK. However, the sample size included a variety of midwives in terms of age, years of experience and roles which allowed insights from a wide range of perspectives and limited potential bias.

The postural analysis study addressed not only the different birth positions but also other midwifery tasks reported to cause MSDs including breast feeding support (Thompson, 2000) and perineal suturing. However, birth pool related tasks were not included in the scope of postural analysis study despite being highly reported by the interviewees, because the pools had previously been evaluated and redesigned (Hignett, 1996).

This study was limited by the absence of real practice observations as the tasks were performed with a model, not a childbearing mother. That might have resulted in missing some factors occurring in real practice such as mothers with minimal cooperation and/or resistance with mothers pushing the midwife away. Moreover, it is possible that the participants did not show the postures used in real practice. However, performing with a model had the advantage of creating a controlled environment, with minimal concerns about mother and baby safety. The presence of an external observer (and cameras) might have distracted the midwives and influenced the patient care activities and safety. Apart from the real practice, other conditions (environment, equipment, staff and organisation) were the same, for example midwives' were wearing their uniforms to reflect any potential activity limitations.

The time required to performance certain tasks was reduced; this was another limitation of the study. However, this is difficult to predict as nature of birth varies depending on the mother and baby. 
Although REBA assessed many aspects of midwifery working tasks, one issue that was not addressed was the slippery or wet surfaced load (baby), which might have affected the holding force or pressure in the body parts.

\section{Conclusion}

This research explored midwives' experiences about work-related MSDs and contributory factors, their level of awareness and support by the organisation about safe practice with an evaluation of the most frequent, extreme and working positions. An in-depth exploration of the main issues through interviews showed that MSDs were often attributed to the physical (working in awkward position, caring for mother and baby at the same time), organisational (longer shift hours, fewer staff, increased work load), staff-related (defensive practice) and patient-related (higher expectation) demands of midwifery care. Manual handling training was found not to be useful for this group. Individual awareness of level of health and safety was high, but the application of such procedures was not possible in a real working environment. The analysis of postures has shown that midwifery working postures have very high to medium risk levels, mostly for the back, neck and upper arms. This indicates that action or further assessment is definitely necessary (with some urgency) to reduce work-related MSDs for midwifery tasks in birth and post-birth. These data, therefore, can guide the development of effective MSDs prevention strategies because they provide a multifactorial approach.

\section{Acknowledgement}

This research did not receive financial support from any funding agencies.

\section{References}

Aronsson, G., Gustafsson, K., Dallner, M., 2000. Sick but yet at work. An empirical study of sickness presenteeism. J. Epidemiol. Community Heal. 54, 502-509.

Aysun, K., Bayram, Ş., 2017. Determining the level and cost of sickness presenteeism among hospital staff in Turkey. Int. J. Occup. Saf. Ergon. 23, 501-509.

Ball, J., Maben, J., Murrells, T., Day, T., Griffiths, P., 2014. 12 - hour shifts : Prevalence, views and impact. 
Bergström, G., Bodin, L., Hagberg, J., Aronsson, G., Josephson, M., 2009. Sickness presenteeism today, sickness absenteeism tomorrow? A prospective study on sickness presenteeism and future sickness absenteeism. J. Occup. Environ. Med. 51, 629-638.

Bierla, I., Huver, B., Richard, S., 2013. New evidence on absenteeism and presenteeism. Int. J. Hum. Resour. Manag. 24, 1536-1550.

Black, C.D., Frost, D., 2011. Health at work-an independent review of sickness absence. The Stationery Office.

Camerino, D., Estryn-Behar, M., Conway, P.M., van Der, B.I.J.M., Hasselhorn, H.-M., 2008. Workrelated factors and violence among nursing staff in the European NEXT study: a longitudinal cohort study. Int. J. Nurs. Stud. 45, 35-50.

CIEHF, 2018. Human Factors for Health and Social Care, White Paper.

Clemes, S.A., Haslam, C.O., Haslam, R.A., 2009. What constitutes effective manual handling training? A systematic review. Occup. Med. (Chic. III). 60, 101-107.

Cumberlege, J., 2016. Better Births. Improving outcomes of maternity services in England. Natl. Matern. Rev. 25.

Dawson, A.P., McLennan, S.N., Schiller, S.D., Jull, G.A., Hodges, P.W., Stewart, S., 2007. Interventions to prevent back pain and back injury in nurses: a systematic review. Occup. Environ. Med. 64(10), 642-650.

Fallentin, N., Kilbom, Å., Viikari-Juntura, E., Wærsted, M., 2000. Evaluation of physical workload standards/guidelines from a Nordic perspective, in: Proceedings of the Human Factors and Ergonomics Society Annual Meeting. SAGE Publications Sage CA: Los Angeles, CA, pp. 6-429.

Gilworth, G., Chamberlain, M.A., Harvey, A., Woodhouse, A., Smith, J., Smyth, M.G., Tennant, A., 2003. Development of a work instability scale for rheumatoid arthritis. Arthritis Care Res. (Hoboken). 49, 349-354.

Griffiths, P., Dall'Ora, C., Simon, M., Ball, J., Lindqvist, R., Rafferty, A.-M., Schoonhoven, L., Tishelman, C., Aiken, L.H., 2014. Nurses' shift length and overtime working in 12 European countries: the association with perceived quality of care and patient safety. Med. Care 52, 975.

Health and Social Care Information Centre, 2015. NHS Sickness Absence Rates - January 2015 to March 2015 and Annual Summary 2009 - 10 to 2014 - 15 1-17.

Health Safety Executive, 2006. Managing shiftwork - Health and safety guidance. HSE books 1-45.

Hignett, S., 2003. Intervention strategies to reduce musculoskeletal injuries associated with handling patients: a systematic review. Occup. Environ. Med. 60, e6-e6.

Hignett, S., 2001. Embedding ergonomics in hospital culture: top-down and bottom-up strategies. Appl. Ergon. 32, 61-69.

Hignett, S., 1996. Manual handling risks in midwifery: identification of risk factors. Br. J. Midwifery 4, 590-596. https://doi.org/10.12968/bjom.1996.4.11.590

Hignett, S., McAtamney, L., 2000. Rapid entire body assessment (REBA). Appl. Ergon. 31, 201-205.

Hildingsson, I., Westlund, K., Wiklund, I., 2013. Burnout in Swedish midwives. Sex. Reprod. Healthc. 4, 87-91.

Hogan, D.A.M., Greiner, B.A., O'Sullivan, L., 2014. The effect of manual handling training on achieving training transfer, employee's behaviour change and subsequent reduction of workrelated musculoskeletal disorders: a systematic review. Ergonomics 57, 93-107.

Koo, T.K., Li, M.Y., 2016. A Guideline of Selecting and Reporting Intraclass Correlation Coefficients for 
Reliability Research. J. Chiropr. Med. 15, 155-163. https://doi.org/10.1016/j.jcm.2016.02.012

Leversidge, A., 2016. Why Midwives Leave - revisited. Midwives 19, 19.

Lipscomb, J.A., Trinkoff, A.M., Geiger-Brown, J., Brady, B., 2002. Work-schedule characteristics and reported musculoskeletal disorders of registered nurses. Scand. J. Work. Environ. Health 394401.

LoBiondo-Wood, G., Haber, J., 2006. Nursing research St. Louis, MO Mosby Elsevier.

Long, M.H., Bogossian, F.E., Johnston, V., 2013a. Functional consequences of work-related spinal musculoskeletal symptoms in a cohort of Australian midwives. Women and Birth 26, e50-e58.

Long, M.H., Bogossian, F.E., Johnston, V., 2013b. Midwives' Experiences of Work-Related Shoulder Musculoskeletal Problems. Int. J. Childbirth 3, 52-64.

Long, M.H., Johnston, V., Bogossian, F., 2012. Work-related upper quadrant musculoskeletal disorders in midwives, nurses and physicians: a systematic review of risk factors and functional consequences. Appl. Ergon. 43, 455-467.

Long, M.H., Johnston, V., Bogossian, F.E., 2013c. Helping women but hurting ourselves? Neck and upper back musculoskeletal symptoms in a cohort of Australian Midwives. Midwifery 29, 359367.

Lundgren, I., Berg, M., Nilsson, C., Olafsdottir, O.A., 2019. Health professionals' perceptions of a midwifery model of woman-centred care implemented on a hospital labour ward. Women and Birth.

Mannion, R., Davies, H., Marshall, M., 2004. Cultures for performance in health care. McGraw-Hill Education (UK).

Mannion, R., Davies, H.T.O., Marshall, M.N., 2005. Cultural characteristics of "high" and "low" performing hospitals. J. Health Organ. Manag. 19, 431-439.

Marshall, F., 2018. Boy, 6, awarded record f37m in NHS compensation after 'catastrophic' brain damage. Telegr.

McDermott, H., Haslam, C., Clemes, S., Williams, C., Haslam, R., 2012. Investigation of manual handling training practices in organisations and beliefs regarding effectiveness. Int. J. Ind. Ergon. 42, 206-211.

NHS Resolution, 2018. Annual report and accounts: 2017/18. London.

Nowotny-Czupryna, O., Naworska, B., Brzęk, A., Nowotny, J., Famuła, A., Kmita, B., Bąk, K., 2012. Professional experience and ergonomic aspects of midwives' work. Int. J. Occup. Med. Environ. Health 25, 265-274.

O'Connell, R., Downe, S., 2009. A metasynthesis of midwives' experience of hospital practice in publicly funded settings: compliance, resistance and authenticity. Health: 13, 589-609.

Okuyucu, K., Gyi, D., Hignett, S., Doshani, A., 2019. Midwives are getting hurt: UK survey of the prevalence and risk factors for developing musculoskeletal symptoms. Midwifery 79, 102546.

Passmore, K., Leung, W.C., 2002. Defensive practice among psychiatrists: a questionnaire survey. Postgrad. Med. J. 78, 671-673.

Plant, M., Coombes, S., 2003. Primary care nurses' attitude to sickness absence: a study. Br. J. Community Nurs. 8, 421-427.

RCM, 2015. State of Maternity Services Report 2015 1-16.

RCOG, 2018. Each Baby Counts: 2018 Progress Report, Royal College of Obstetricians and Gynaecologists. 
RCOG, 2016. Each Baby Counts: key messages from 2015, Royal College of Obstetricians and Gynaecologists. London.

Robson, C., McCartan, K., 2016. Real world research. John Wiley \& Sons.

Rogers, A.E., Hwang, W.-T., Scott, L.D., Aiken, L.H., Dinges, D.F., 2004. The working hours of hospital staff nurses and patient safety. Health Aff. 23, 202-212.

Russo, A., Murphy, C., Lessoway, V., Berkowitz, J., 2002. The prevalence of musculoskeletal symptoms among British Columbia sonographers. Appl. Ergon. 33, 385-393.

Scott, T., Mannion, R., Marshall, M., Davies, H., 2003. Does organisational culture influence health care performance? A review of the evidence. J. Health Serv. Res. Policy 8, 105-117.

Skagen, K., Collins, A.M., 2016. The consequences of sickness presenteeism on health and wellbeing over time: a systematic review. Soc. Sci. Med. 161, 169-177.

Smedley, J., Inskip, H., Trevelyan, F., Buckle, P., Cooper, C., Coggon, D., 2003. Risk factors for incident neck and shoulder pain in hospital nurses. Occup. Environ. Med. 60, 864-869.

Smith, D.R., Wei, N., Zhang, Y.-J., Wang, R.-S., 2006. Musculoskeletal complaints and psychosocial risk factors among physicians in mainland China. Int. J. Ind. Ergon. 36, 599-603.

Surtees, R., 2010. 'Everybody expects the perfect baby... and perfect labour... and so you have to protect yourself': discourses of defence in midwifery practice in Aotearoa/New Zealand. Nurs. Inq. 17, 82-92.

Symon, A., 2000. Litigation and defensive clinical practice: quantifying the problem. Midwifery 16, 814.

Szeto, G.P.Y., Ho, P., Ting, A.C.W., Poon, J.T.C., Cheng, S.W.K., Tsang, R.C.C., 2009. Work-related musculoskeletal symptoms in surgeons. J. Occup. Rehabil. 19, 175-184.

Thompson, E., 2000. Safer birthing positions. A choice for mother and midwife. The Column 12, 1722.

Tveten, K.M., Morken, T., 2015. Decision-making in job attendance within health care-a qualitative study. Occup. Med. (Chic. III). 66, 247-251.

Van Mechelen, W., Hlobil, H., Kemper, H.C.G., 1992. Incidence, severity, aetiology and prevention of sports injuries. Sport. Med. 14, 82-99.

Vanwonterghem, K., Yoopat, P., Maes, C., 2012. Musculoskeletal disorders: a new approach. Work 41, 2293-2298.

Verbeek, J.H., Martimo, K., Karppinen, J., Kuijer, P.P., Viikari-Juntura, E., Takala, E., 2011. Manual material handling advice and assistive devices for preventing and treating back pain in workers.

Waters, T.R., 2010. Introduction to ergonomics for healthcare workers. Rehabil. Nurs. 35, 185-191.

Whysall, Z., Bowden, J., Hewitt, M., 2018. Sickness presenteeism: measurement and management challenges. Ergonomics 61, 341-354.

Wilson, J.R., Sharples, S., 2015. Evaluation of human work. CRC press.

Yazdani, A., Neumann, W.P., Imbeau, D., Bigelow, P., Pagell, M., Wells, R., 2015. Prevention of musculoskeletal disorders within management systems: A scoping review of practices, approaches, and techniques. Appl. Ergon. 51, 255-262. 


\section{Appendix 1}

\section{Questions for the study of 'Work-related musculoskeletal injuries amongst midwives'}

Musculoskeletal injuries are injuries and dysfunction of the muscles, tendons, ligaments, nerves etc. in different body parts. Work-related musculoskeletal injuries are problems caused by the work activities and environment.

Age:

Age at first symptom:

Currently workplace and position:

Year of experience:

Work pattern:

1. Can you tell me more about your injury?

- How did it occur?

- Do you think it is work related?

- How did you manage?

- Did you report?

- What options have you considered? Let them list (e.g., self-management, seen by

- GP, seen by PT /OT)

- How was that decision made?

- Have you got benefit of it?

2. A recent survey study showed that the prevalence of musculoskeletal problems in midwives is very high. Considering the regulations and actions taken especially in $80 \mathrm{~s} / 90$ s, why do you think it is still very high?

- What do you think has changed since last 20-30 years that might contribute to the injuries in midwifery?

3. Another result came out from the survey study is that the younger midwives who have less experience in midwifery are more likely to report musculoskeletal discomforts and pain. What do you think the reasons are behind that result?

- How do you think ageing / having more experience affect having musculoskeletal problems?

4. Do you think anything in your workplace contribute in occurrence of injuries (environment, equipment)?

- In case of any work-related musculoskeletal injury, how does the organisation work?

- Are there any actions taken such as adjustments in working place/conditions or so on?

5. Do you think midwives know their rights in an organisation?

6. Is there any concern for complaints among midwives? If yes, why?

- Do you think that concern influences on their practice? If yes, how?

7. How do you protect yourself from getting WRMSD?

- In terms of education and training, are midwives thought enough how to protect themselves to avoid musculoskeletal injuries at work?

- What do you think about manual handling trainings?

- Do you think over the years manual handling training has changed? 
8. Can you tell me what the consequences of your injury were in terms of the care you provided?

- Have you ever felt that you could not support the mother?

- Have you influenced the mothers' choices for the delivery based on the number of options given (e.g., birthing pool, epidural)?'

- What is your coping strategy, if the mother wants to deliver in a particular position that you don't feel able to support her?'

9. What additional suggestions can you give to reduce or prevent musculoskeletal injuries experienced by midwives?

10. The following study will analyse the most frequent and extreme work related challenges in midwifery to find out the level of exposure on musculoskeletal system. I would like to ask your opinion, what are the most frequent and extreme work related challenges in midwifery?

- Can you please rate them from 0 to 10, with 0 representing 'not a challenge at all' and 10 representing 'extremely challenging'?

Thank you very much for your contribution. 